\title{
An Artificial Neural Network approach for parametric study on Welding Defect Classification
}

mei po HO ( $\square$ mabel.ho@polyu.edu.hk)

The Hong Kong Polytechnic University https://orcid.org/0000-0002-8885-8428

\section{Wing-kit NGAI}

The Hong Kong Polytechnic University

\section{Tai-wing CHAN}

The Hong Kong Polytechnic University

\section{Hon-wah WAI}

The Hong Kong Polytechnic University

\section{Research Article}

Keywords: ANN, MIG welding, defect classification, welding parameters, Ultrasonic inspection

Posted Date: March 1st, 2021

DOl: https://doi.org/10.21203/rs.3.rs-250028/v1

License: (c) (1) This work is licensed under a Creative Commons Attribution 4.0 International License. Read Full License

Version of Record: A version of this preprint was published at The International Journal of Advanced Manufacturing Technology on February 2 nd, 2022. See the published version at https://doi.org/10.1007/s00170-022-08700-8. 


\section{Abstract}

In this paper, a welding defect prediction model has been developed and investigated through training an Artificial Neural Network (ANN) based model. The input data were three welding process measurements (Welding current, Travel speed, and Protective gas Flow). The output data were non-destructive test results of respective weldments on four defect types (Underfill, Lack of penetration, Incomplete fusion, and Porosity) to ensure the consistency of the welding following the designed parameters, all data were obtained from 289 specimens produced by an automated MIG welding manufacturing system. The 2stages model with 13 inputs, hidden layers with 80-100 neurons, 4 outputs. the outputs were used to evaluate the classification accuracy in the confusion matrix for the prediction of weld quality. A further 73 specimens were used to test the accuracy of the trained ANN model. The model achieved $85 \%$ accuracy.

\section{Introduction}

MIG welding is one of the most widely used processes in shipbuilding, automobile manufacturing, and especially for section prefabrication in construction industry. MIG using externally supplied gas or gas mixture as shielding to minimize contact of the molten metal with air as the constituent of air such oxygen and nitrogen are reactive with metal in high temperature seriously damage the strength and toughness of the weld joint. There is a continuous market demand for a master welder in different industries. Application of a welding robot would alleviate the situation on the problem raised by the heavy workload of the welders and man-power limitation. However, welded joint inspection is still needed to be undertaken by the skilled welder/ inspector.

Both destructive testing (DT) and nondestructive testing (NDT) are used for quality control of the welding in the construction industry. Destructive testing of welds includes macro etch testing, fillet weld break test, transverse tension test and guided bend test. For example, visual surface inspection is needed for every welded joint, ultrasonic inspection and eddy current testing are employed to test on-site. Other testing such as acoustic emission, magnetic particle inspection and cross-sectional inspection through an optical microscope and radiographic imaging are used frequently as well in the laboratory for welded samples. These tests are known as a manual operation which is time-consuming and labor-intensive. With the increase of application of robotic welding, a high requirement of automated, systematic and speedy sample quality checking is put forward.

Although the quality of welding could be estimated or modeled by welding input parameters, welding processes are non-linear complex systems with multiple input/output correlated parameters. Owing to this, the experiences for the determination of critical parameters play a very significant role in monitoring the quality of the final welded structure. Artificial Intelligence for interconnecting the elements with highly hierarchical design, could be applied as a tool to analyze the welding parameters for incorporating knowledge in welding technology, to eliminate some tests, and thus to achieve faster defect classification.

Several researchers have studied the optimization and prediction of MIG welding process parameters. Joseph I. Achebo et al. and Amberar et al, optimize the input parameters including welding current, travel 
speed and other variables against mechanical properties through the Taguchi method $[2,4]$.

It is one of today's most rapidly growing technical fields, lying at the intersection of computer science and statistics, and the core of artificial intelligence and data science [22-23]. An ANN is a learning system based on a computational technique, which attempts to simulate human intuition in making decisions and drawing conclusions when presented with complex, noisy, irrelevant, and partial information [6-7]. An ANN is a data-driven self-adaptive method and able to approximate any reasonable function arbitrarily well. The ability of an ANN to learn and generalize the behaviour of any complex and non-linear process makes it a powerful modeling tool [8]. Haken et al. predicted the tensile strength, impact strength, elongation and hardness from welding input variables gas mixtures through artificial neural networks [1]. Amit Kumar et al. applied the ANN to optimize the MIG welding parameters welding current, voltage and travel speed through output parameters ultimate tensile strength [3]. Patel et al. developed an ANN model to predict the weld height through the input variables welding current, welding speed and gas flow rate [5].

There are limited publication has been found on discussing the relationship between the current (taking the fluctuation of the current during the welding into account, welding speed and gas flow and the welding defect classification. In this paper, an ANN model is proposed for prediction of welding defect and optimization for the welding parameters in MIG welding to prevent the domination of the current to gas flow and welding speed, a two-stage network has been designed for further investigation.

\section{Methodology}

To control the welding quality, the automated MIG specimen welding system was designed and developed. Figure 1 is the automated MIG welding testbed. The main components in the system include (1) an Industrial MIG Welder with calibrated control of filler feed rate, voltage, and current; (2) An automated modular drive system to move the welding torch of (1) at preset velocity; (3) A specimen fixture is designed to position the parts for welding and secure the geometry of the product parts; (4) Two welding cameras to capturing the video image of weld pool from two field-of-views; (5) A gas flow regulator to control the shielding-gas flow at preset rate; (6) A process control and data logging system to control the operation of different components and capture the test data for further analysis.

The specimen geometry (Figure 2) and preparation are conformed to ISO 9692-1 "Welding and allied processes - Types of joint preparation - Part 1: Manual metal arc welding, gas-shielded metal arc welding, gas welding, TIG welding and beam welding of steels". The material is $50 \mathrm{~mm} \times 140 \mathrm{~mm} \times 6 \mathrm{~mm}$ mild steel plate with one backing strip $25 \mathrm{~mm} \times 140 \mathrm{~mm} \times 6 \mathrm{~mm}$ [16].

A design of experiments (DOE) was created to vary three input process parameters: current, welding speed and gas flow. A total of 362 samples were prepared and tested for ANN training and testing. Table 1 shows the parameters used in this study and its range. The data logger recorded the current at a rate of 1 $\mathrm{Hz}$ and the data were divided into 11 groups. The mean of each group of data was obtained as the input 
variable for Al training. The travel speed was filed, and gas flow was measured through a gas flow regulator.

After the welding, a visual inspection is conducted to evaluate the weld bead conditions. Wiki-scan was used to visualize the geometry data including bead width, height through laser sensor technology to ensure the data conformity. Using AWS D1.1 chapter 6 [18] and IS016811:2012 [19] as a reference, the SONATEST VEO +16:64 PAUT flaw detector was used with Olympus phased-array probe 5MHz, 16 elements and $0.6 \mathrm{~mm}$ pitch probe for UT nondestructive testing. The specimens were tested through angle beam examination (transverse wave) are were used. Standard small-footprint wedge SA10P-N55S was applied to provide a specific angle and transducer designed for weld joint inspection for detecting the flaws and sizing of weld defect. Sectional scan images in $10 \mathrm{~mm}$ were captured and stored for defect recognition. To control the scanning distance and minimize the extraneous damping, a scanning fixture was tailor-made and the design. It takes the groove scan plus an extra $6 \mathrm{~dB}$ as reference $\mathrm{dB}$. Currently, 45 $\mathrm{dB}$ is adjusted to perform the ultrasonic testing of the specimens. The procedures of the ultrasonic inspection were guided by ISO 13588:2019 (E) [17]. Due to the risk of delayed cracking, a period of at least $48 \mathrm{~h}$ is generally required before the final inspection is made of as-welded fabrications. Fixed angles at fixed probe position to weld were performed on both sides of the weld.

\section{Results And Discussion}

According to ISO 17635:2016 [20], welded joints should typically be tested and evaluated by visual testing, before testing for internal discontinuities. The weld bead profile parameters such as width and height were measured by WiKi-Scan (laser scan), to specify the quality level of imperfections (BS EN ISO 5817:2014) [21]. The weld defects including underfill and undercut were verified by observation and laser scanning. Other surfaces defects such as burn through, and spatter were checked through visual inspection. Figures $3(a-d)$ show the examples of the clean weld and the welded joint with defects.

For each specimen, 22 ultrasonic images in total will be captured from both the left and right sides of the welding. Each image corresponds to a specific section of the welding with a length of $10 \mathrm{~mm}$. Figure 4 (ad) are the examples of ultrasonic images captured. For simplifying the data formatting, "2" for very poor defect, " 1 " for fair defect and " 0 " for the proper and clean weld. The severity is based on the intensity of imperfection. Then, it will have 22 imperfections values for each of the specimens and here comes the methodology of deciding whether the whole sample is defective or clean.

The acceptance of weld discontinuity is determined based on its indication rating and its length, following ISO 17640 [21] and AWS D1.1/D1.1M. Four levels of acceptances including large, medium, small, minor are applied to classify the discontinuity. Those discontinuities classified as class A to class D referred to large, medium, small and minor are necessary to be recorded in the test report. Table 3 is the indication of imperfections in samples.

Data including welding current captured through real-time data logging and defect classified through laser scanning, visual and UT inspection is pro-processed. [1x13] array was created for each specimen 
and the welding process parameters including current, welding speed and gas flow were assigned to an element in an array.

The collection of the data was completed based on two data logs: machine log data and route card data. Welding process parameters as inputs datasets are matched with the UT testing result as outputs dataset for the network by every section in $10 \mathrm{~mm}$. Welding current was captured by data logger with $0.1 \mathrm{~s}$. Therefore, a simple python script was designed that will help us to manage all input dataset and output datasets. Basically, the program script was executed to extract all data in ithe nput dataset and the welding current values were stored for each sample individually. After that, an array splitter was used to partition the current data of each sample into 11 arrays. The arithmetic mean of each array was measured and as the first 11 elements of the input dataset [1x13] array of each specimen.

Consequently, the welding speed value for every sample is extracted from the microcontroller log and assigned as the 12th element of the [1x13] array. The amount of gas flow for each sample is captured from the welding route card and assigned as the $13^{\text {th }}$ elements of the [1x13] array analogically. To sum up, there are 13 values in the array as an input data structure with 11 data is the currents which are the arithmetic mean from the welder logger. 1 data is the traveling speed which is recorded by the microcontroller logger and encoder. And the gas flow is recorded by the analogical input. Weld defect data were obtained from surface and ultrasonic inspection and assigned by Python script as the known output values as a vector from a collected data set. Meanwhile, the outputs are classified as 1 or 0 for such defects as incomplete penetration, incomplete fusion, porosity, and underfill.

The two-stage ANN model is developed and depicted in figure 5. The ANN model consists of an input layer, a hidden layer and an output layer. The input is multiplied by the weight associated with the synapse connecting the input to the current neuron. As there are 11 current inputs, 1 welding speed input and 1 gas flow input, the data of the current may dominate the prediction. therefore, to balance the importance of the 3 parameters, two stages ANN model has been developed. the model was separated into 3 parts including "right input", "left input" and "concatenate". Right input supports processing the currents data to obtain a result between " 0 " to " 1 ". Left input supports processing the gas flow and welding speed data to obtain two outputs the outputs are the number between " 0 " to " 1 ". three outputs were obtained as input for prediction on welding defect.

Network optimization can be conducted to optimize network hyperparameters for improving network performance and accuracy. The final output results obtained from the neural network are the probabilities of the outcomes. The overall hyperparameters of ANN is depicted in table 2.

Neural networks apply a non-linear activation function to support the network to learn complex data and provide accurate predictions. Using leaky ReLU will provide a faster and higher overall accuracy training which is suitable for training with many input neuron models. Tanh's deviate is steeper, which means it can obtain more value and such function is more efficient because it has a wider range for faster learning and grading [23]. The sigmoid function is commonly used especially for the case in which output is multiclass classification. using the sigmoid activation in the second stage produces the outputs with the result 
in $[0,1]$. The output of the network ranged between 0 and 1.0 means that the corresponding specimen with "no defect" and 1 means that corresponding specimen with "defect'. using stochastic gradient descent (SGD) optimization for the optimal solution. the loss function was used is binary crossentropy. to control the number of training samples to work through, the batch size was set to 64 . The initial epochs were set to 1200 and finally set to base on the Training and validation accuracy per epoch in the finalized ANN model depicted. Randomly draw samples from a Gaussian distribution is used for samples drawing.

The results of the ANN model are shown in Table 5. The ANN model correctly predicts the positive class is $54.5 \%$ (True positive, i.e. sample with the defect is classified as defective). The model incorrectly predicts the positive class is $8.9 \%$ (false positive, ie, sample without defect is classified as defective). The model correctly predicts the negative class is $30.1 \%$ (True negative, the clean weld is classified as nondefective). The model incorrectly predicts the negative class is $6.5 \%$ (false negative, ie, a sample with a defect is classified as non-defective). The finalized ANN computing approach had $84.589 \%$ accuracy $(54.5+30.1 \%)$. The specificity (True negative rate) is $77.193 \%$. The sensitivity (True positive rate) is $89.326 \%$.

Figures 6 and 7 are the learning curves of the finalized ANN model. The curves indicate that the model is not either underfit or overfit. Figure 6 shows that the training and validation datasets are suitably representative of the problem domain. Figure 7 is the training curve and the validation loss for the ANN model with Binary crossentropy under 800 epochs. The training was stopped at 750 epochs once the model performance stops improving the dataset and to avoid overfitting. Figure 7 depicted the tendency of the model to be overfitting. overfitting refers to fitting a more flexible model requires estimating the parameters. More complex models can lead to a phenomenon known as overfitting, which essentially means following the errors, or noise, too closely [9-11]. Using cross validation and early stopping can avoid overfitting. compared with cross-validation, $\mathrm{K}$ - fold cross- validation (CV) is more powerful which was employed to estimate the out-of-sample error by splitting the dataset into K-fold. the test error was thus calculated, and averaged o K runs, to yield the final out-of-sample error [12-14]. another method is called "early stopping (ES) which is a regularization to avoid overfitting [15]. It guides as to when the classifier should end before the overfitting begins. commonly, the training is stopped until the validation error is higher than its last value [15].

A user interface has been designed and tailor-made to support the application of the Al system. The first function of the interface is generating the optimized welding parameters using the developed Al system. Figure 8 shows a control panel prototype created for generating the optimized welding parameters based on input either current, speed or flow rate. For example, input the current will generate the speed and flow based on the Al prediction. Another function of the interface using the developed Al system to classify the potential defects which may be occurred in the welded pieces. The user interface shown in figure 9 , current, speed and flow rate must be entered by the user to obtain the Al-based welding defect prediction.

\section{Conclusion}


In this paper, a welding defect prediction model has been developed and investigated through training an Artificial Neural Network (ANN) based model. The input data were three welding process measurements (Welding current, Travel speed, and Protective gas Flow). The output data were nondestructive test results of respective weldments on four defect types (Underfill, Lack of penetration, Incomplete fusion, and Porosity) to ensure the consistency of the welding following the designed parameters, all data were obtained from 289 specimens produced by an automated MIG welding manufacturing system. The 2-stages model with 13 inputs, hidden layers with 80-100 neurons, 4 outputs. the outputs were used to evaluate the classification accuracy in the confusion matrix for the prediction of weld quality. A further 73 specimens were used to test the accuracy of the trained ANN model. The model achieved $85 \%$ accuracy.

\section{Declarations}

\section{Funding}

The content of this paper is fully / partially funded by the Chinese National Engineering Research Centre for Steel Construction (Hong Kong Branch) at the Hong Kong Polytechnic University.

Conflicts of interest/Competing interests

There is no conflict of interest in this work.

Availability of data and material

Data available on request from the authors. The data that support the findings of this study are available from the corresponding author, Mei-Po, Ho, upon reasonable request.

Consent to participate/ Publish

The manuscript has not been published previously (partly or in full). No data has been fabricated or manipulated and no data, text, or theories by others are presented as if they were the author's own. Proper acknowledgements to other works have been given. Consent to submit has been received explicitly from all co-authors. Authors whose names appear on the submission have contributed sufficiently to the scientific work and therefore share collective responsibility and accountability for the results.

Compliance with ethical standard

This research does not involve any human or animal participant. All professional ethics have been followed. The manuscript has not been submitted to other journal for simultaneous consideration.

\section{Acknowledge}

This is to acknowledge that the project leading to publication of this paper is fully / partially funded by the Chinese National Engineering Research Centre for Steel Construction (Hong Kong Branch) at the Hong 
Kong Polytechnic University.

\section{References}

[1] Ates, H. (2007). Prediction of gas metal arc welding parameters based on artificial neural networks. Materials \& design, 28(7), 2015-2023.

[2] Achebo, J. I. (2011, July). Optimization of GMAW protocols and parameters for improving weld strength quality applying the Taguchi method. In Proceedings of the world congress on engineering (Vol. 1, pp. 6-8).

[3] Kumar, A., Jadoun, R. S., \& Bist, A. S. INTERNATIONAL JOURNAL OF ENGINEERING SCIENCES \& RESEARCH TECHNOLOGY Optimization of MIG welding parameters using Artificial Neural Network (ANN) and Genetic Algorithm (GA).

[4] Ambekar, S. D., \& Wadhokar, S. R. (2015). Parametric optimization of gas metal arc welding process by using Taguchi method on stainless steel AISI 410. International Journal of Research in Modern Engineering and Emerging Technology, 3(1), 1-9.

[5] Patel, T., Sheth, S., Modi, B. S., \& Patel, P. (2015, January). Experimental investigation and comparison of regression model and artificial neural network to predict weld height in MIG welding for dual plate check valve. In International conference on ADVANCES IN MATERIALS AND PRODUCT DESIGN AMPD.

[6] Modelling of lead adsorption from industrial sludge leachate on red mud by using RSM and ANN.

[7] Youssefi, S., Emam-Djomeh, Z., \& Mousavi, S. M. (2009). Comparison of artificial neural network (ANN) and response surface methodology (RSM) in the prediction of quality parameters of spray-dried pomegranate juice. Drying Technology, 27(7-8), 910-917.

[8] Geyikçi, F., Kılıç, E., Çoruh, S., \& Elevli, S. (2012). Modelling of lead adsorption from industrial sludge leachate on red mud by using RSM and ANN. Chemical Engineering Journal, 183, 53-59.

[9] Van der Aalst, W. M., Rubin, V., Verbeek, H. M. W., van Dongen, B. F., Kindler, E., \& Günther, C. W. (2010). Process mining: a two-step approach to balance between underfitting and overfitting. Software \& Systems Modeling, 9(1), 87. (overfitting and underfitting)

[10] Jabbar, H., \& Khan, R. Z. (2015). Methods to avoid over-fitting and under-fitting in supervised machine learning (comparative study). Computer Science, Communication and Instrumentation Devices, 163-172. (overfitting and underfitting)

[11] James, G., Witten, D., Hastie, T., \& Tibshirani, R. (2013). An introduction to statistical learning (Vol. 112, p. 18). New York: springer. 
[12] Esmaeelzadeh, S. R., Adib, A., \& Alahdin, S. (2015). Long-term streamflow forecasts by Adaptive Neuro-Fuzzy Inference System using satellite images and K-fold cross-validation (Case study: Dez, Iran). KSCE Journal of Civil Engineering, 19(7), 2298-2306.

[13] Kolus, A., Imbeau, D., Dubé, P. A., \& Dubeau, D. (2015). Adaptive neuro-fuzzy inference systems with kfold cross-validation for energy expenditure predictions based on heart rate. Applied ergonomics, 50, 6878.

[14] Zhang, Y. D., Hou, X. X., Chen, Y., Chen, H., Yang, M., Yang, J., \& Wang, S. H. (2018). Voxelwise detection of cerebral microbleed in CADASIL patients by leaky rectified linear unit and early stopping. Multimedia Tools and Applications, 77(17), 21825-21845.

[15] Famouri, M., Taheri, M., \& Azimifar, Z. (2015). Fast linear svm validation based on early stopping in iterative learning. International Journal Of Pattern Recognition And Artificial Intelligence, 29(08), 1551013.

[16] ISO, E. 9692-1 (2003) Welding and allied processes-Recommendations for joint preparation-Part1: Manual metal-arc welding, gas shielded metal-arc welding, gas welding, TIG welding and beam welding of steels.

[17] ISO 13588:2019. Non-destructive testing of welds - Ultrasonic testing - Use of automated phased array technology.

[18] Furr, P. A. (2014, October). AWS D1. 1 and D1. 5 Phased Array Examination. In ASNT Annual Conference 2014 (pp. 52-58).

[19] ISO 16811:2012. Non-destructive testing - Ultrasonic testing - Sensitivity and range setting [20] EN ISO 17635:2016, Non-destructive testing of welds - General rules for metallic materials [21] ISO, B. 17640: 2010,". Non-destructive testing of welds-Ultrasonic testing-Techniques, testing levels, and assessment.

[22] Maheshwary, S., Ganguly, S., \& Pudi, V. (2017). Deep secure: A fast and simple neural network based approach for user authentication and identification via keystroke dynamics. In IWAISe: First International Workshop on Artificial Intelligence in Security (p. 59).

[23] Jordan, M. I., \& Mitchell, T. M. (2015). Machine learning: Trends, perspectives, and prospects. Science, 349(6245), 255-260.

\section{Tables}

'able 1 - The variances and the ranges of randomized samples for training 


\section{ype of Variances}

Unit Lower range Upper range

\begin{tabular}{cccc}
\hline Current & A & 180 & 300 \\
Speed & In/min & 6 & 18 \\
\hline Gas Flow & l/min & 3 & 18
\end{tabular}

Table 2- Hyperparameters of ANN.

\begin{tabular}{|c|c|}
\hline Parameters & Setting \\
\hline \multirow[t]{4}{*}{ Activation function } & $1^{\text {st }}$ stage \\
\hline & Left input: Tanh \\
\hline & Right input: leaky ReLU \\
\hline & $2^{\text {nd }}$ stage \\
\hline \multicolumn{2}{|c|}{ c. Concatenate: sigmoid } \\
\hline Optimization function & Stochastic gradient descent (SGD) \\
\hline Loss function & Binary crossentropy \\
\hline Samples drawing & Randomly draw samples from a Gaussian distribution \\
\hline Batch size & 64 \\
\hline Epochs & $\begin{array}{l}1200 \text { (Initial setting) } \\
\text { stop at } 750\end{array}$ \\
\hline Learning rate $(\mathrm{LR})$ & 0.9 \\
\hline Weight initialization & Random normal \\
\hline \multirow[t]{4}{*}{ Dropout rate } & $1^{\text {st }}$ stage \\
\hline & Left input: 0.4 \\
\hline & Right input: 0 \\
\hline & $2^{\text {nd }}$ stage \\
\hline d. $\mathrm{Cc}$ & atenate: 0 \\
\hline
\end{tabular}

Table 3 - The indication of imperfections in samples. 
ik of penetration

butt welds: $\mathrm{h} \leq 0,4 \mathrm{~s}$, but max. $4 \mathrm{~mm}$

Quality levels D in ISO 5817

complete fusion

butt welds: $\mathrm{h} \leq 0,4 \mathrm{~s}$, but max. $4 \mathrm{~mm}$

Quality levels D in ISO 5817

Porosity

$\mathrm{h} \leq 0,4 \mathrm{~s}$, but max. $4 \mathrm{~mm}$ or $\mathrm{l} \leq \mathrm{s}$, but

$\max .75 \mathrm{~mm}$

Quality levels D in ISO

5817

Table 4 - The input data format in network.

\section{Array}

\begin{tabular}{cccc}
\hline Item & Current & Speed & Gas flow \\
\hline $\begin{array}{c}\text { Element } \\
\text { Unit }\end{array}$ & $1-11$ & 12 & 13 \\
source & A & In arithmetic mean of welder & Microcontroller logger by \\
& logger & encoder & Analogically \\
input
\end{tabular}

Table 5 - The confusion matrix of finalized ANN model.

Final ANN model

\begin{tabular}{ccc}
\hline Confusion Matrix & $54.5 \%$ & $6.5 \%$ \\
& $\begin{array}{c}\text { (True positive) } \\
\text { (False negative) }\end{array}$ & $\begin{array}{c}\text { (F.9\% } \\
\text { (False positive) }\end{array}$ \\
(True negative) \\
Sensitivity & \multicolumn{2}{c}{89.326} \\
Specificity & \multicolumn{2}{c}{77.193} \\
Accuracy & \multicolumn{2}{c}{84.589}
\end{tabular}

\section{Figures}




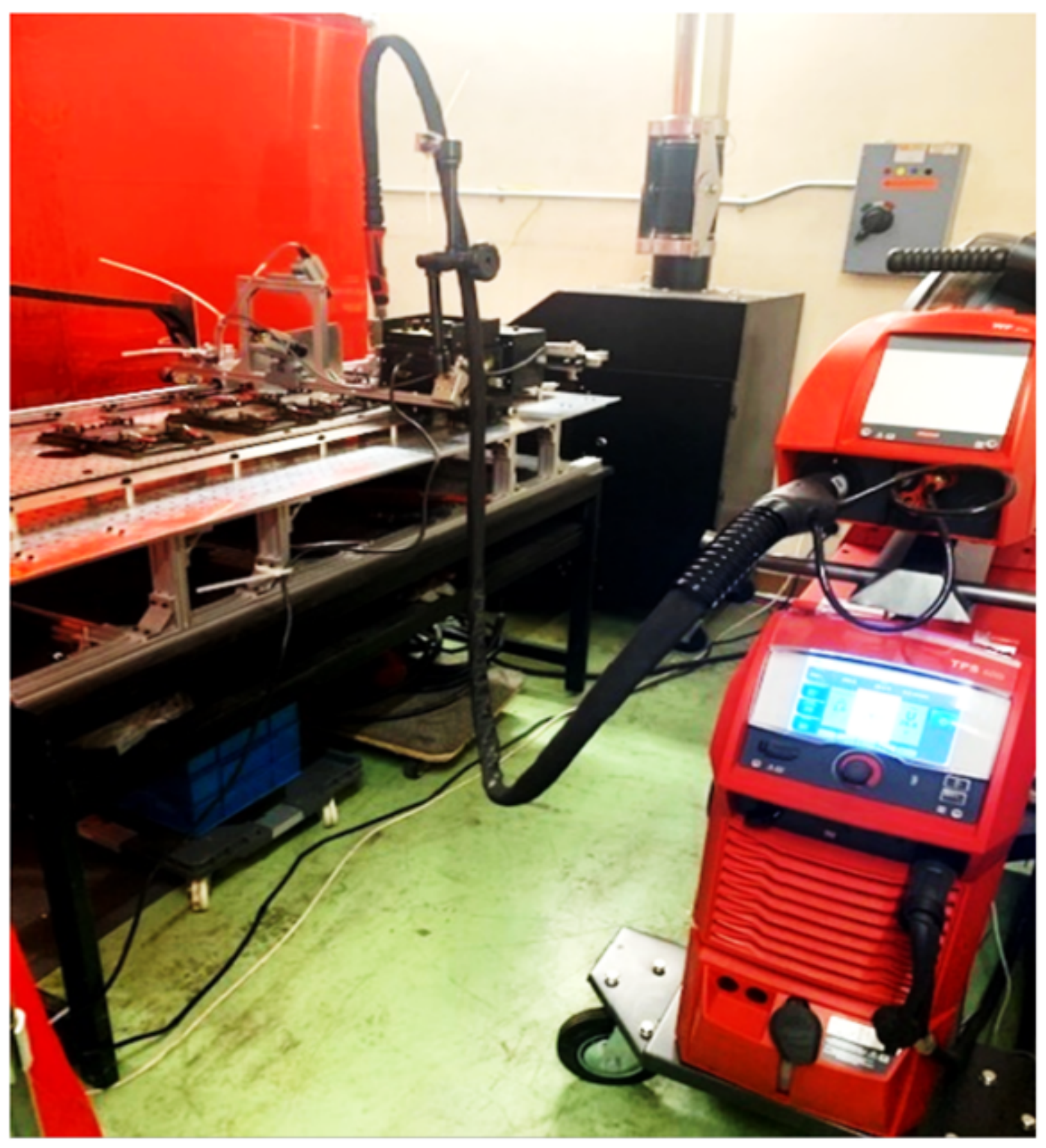

Figure 1

Automated MIG welding manufacturing system. 


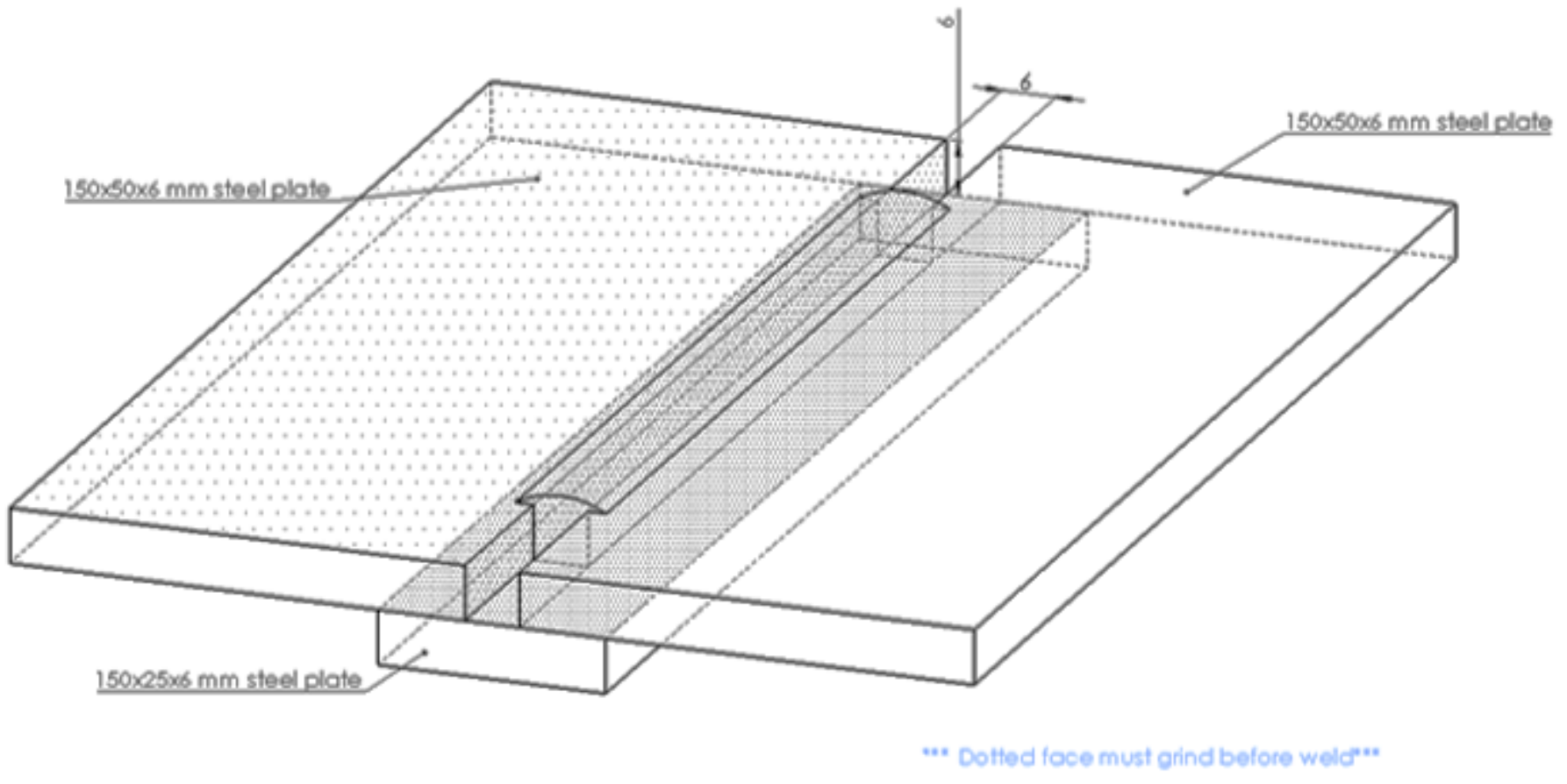

Figure 2

specimen geometry and preparation according to ISO 9692-1 


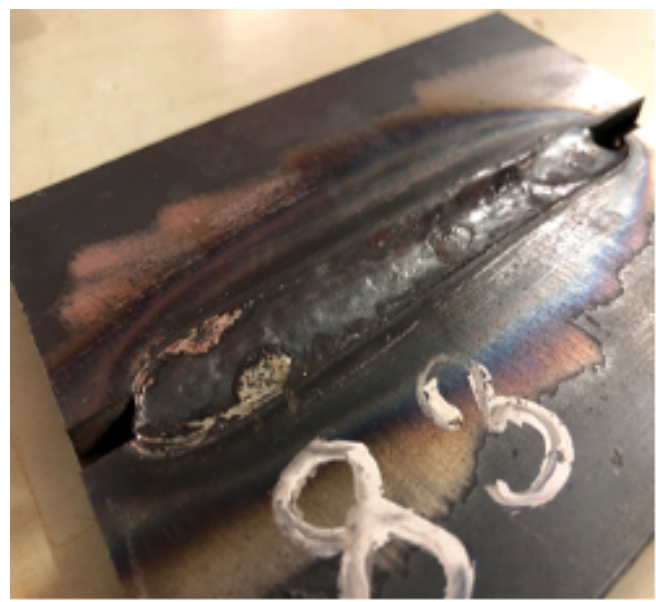

a

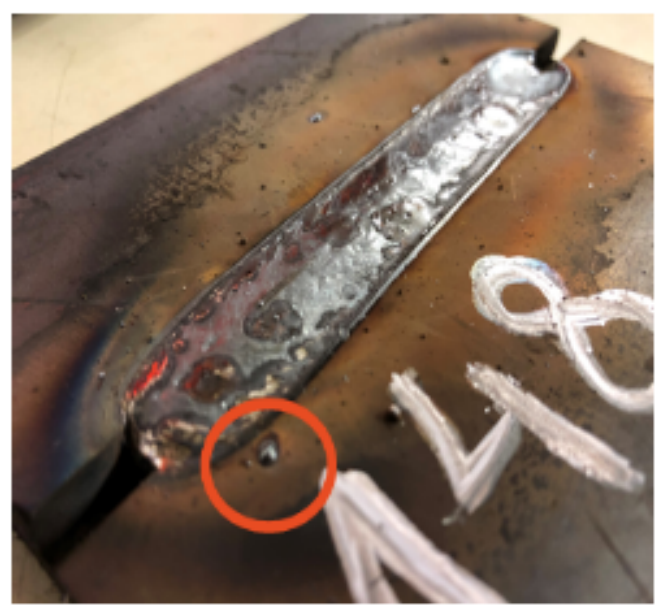

$\mathrm{c}$

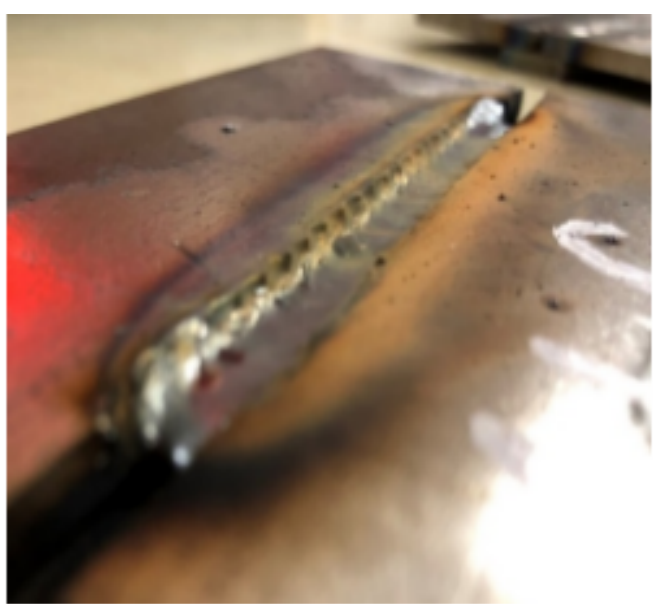

$\mathrm{b}$

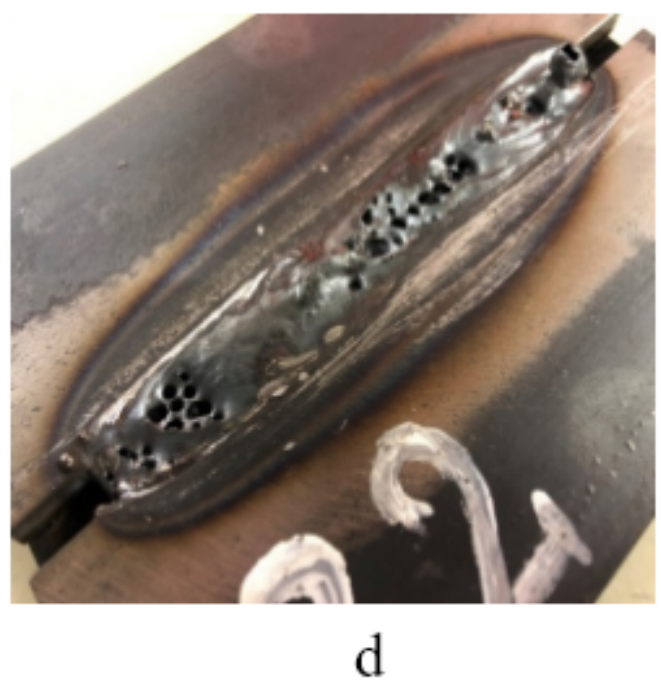

\section{Figure 3}

Welded specimens with (a) no visual defects (b) under fill (c) weld spatter and (d) surface porosity. 


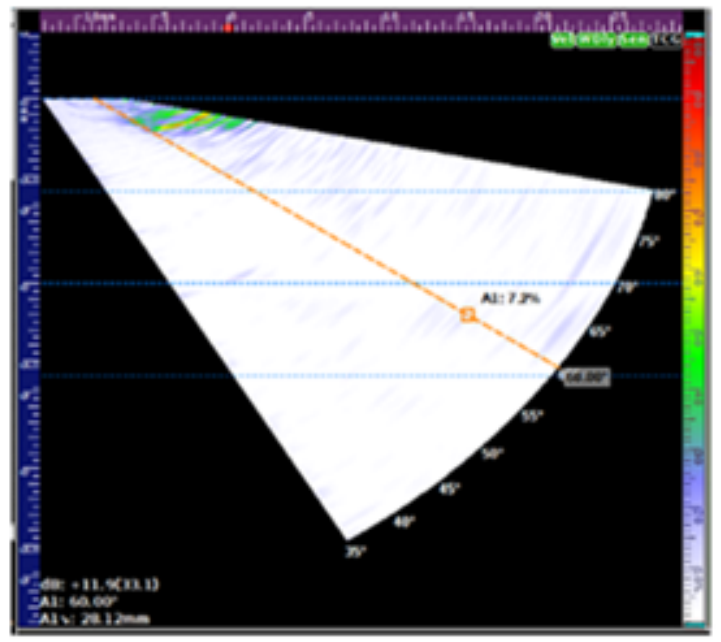

a

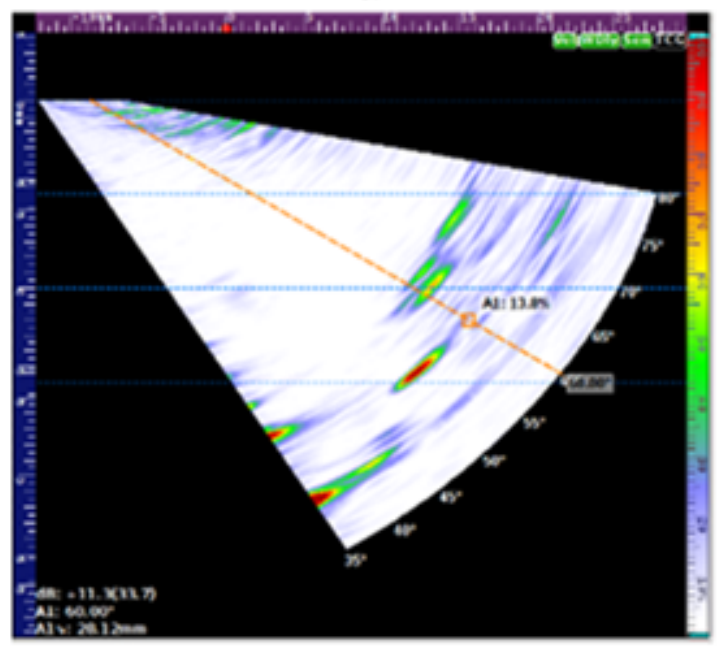

C

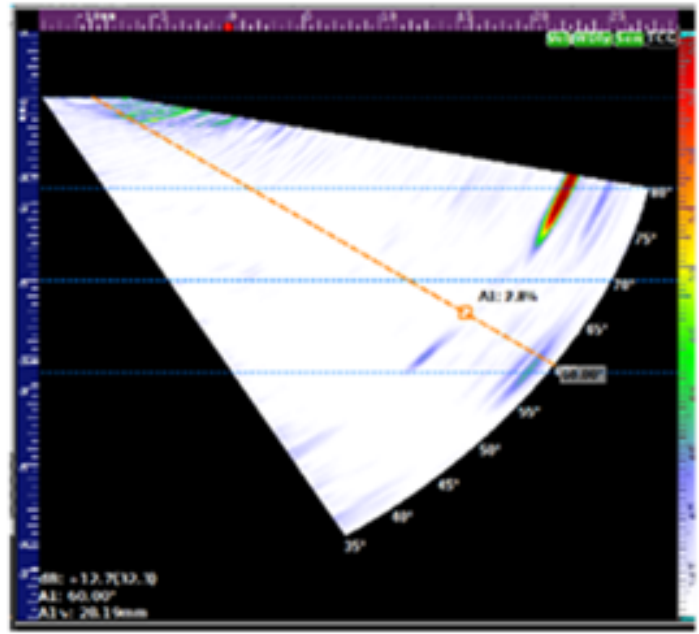

b

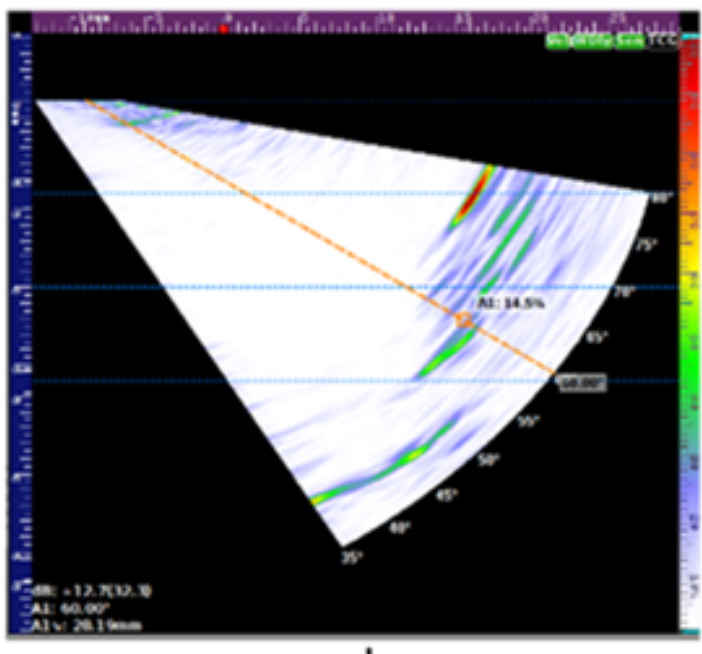

d

\section{Figure 4}

Welded specimens with (a) no visual defects (b) Lack of penetration (c) Incomplete fusion and (d) porosity. 


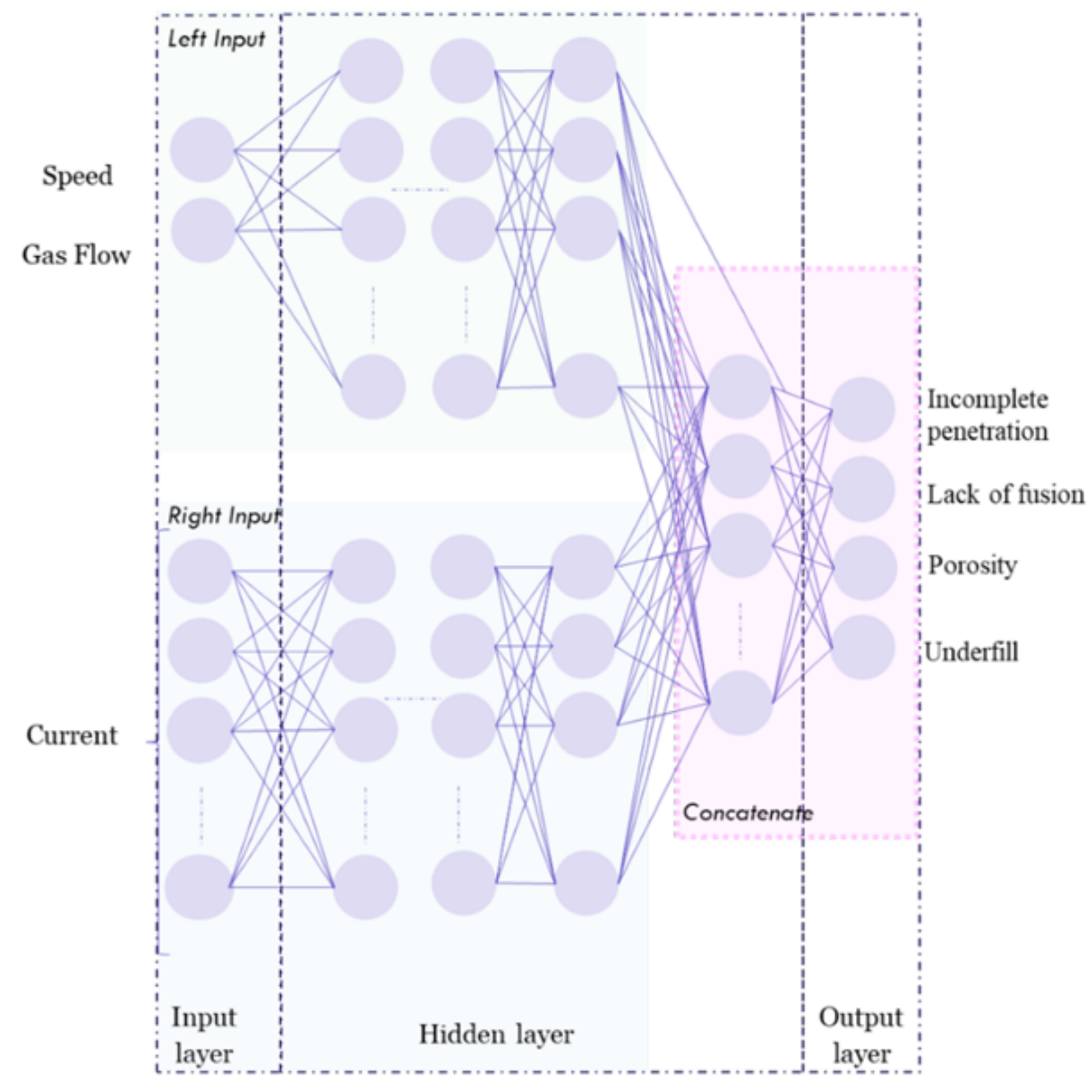

Figure 5

Developed 2-stage ANN architecture 


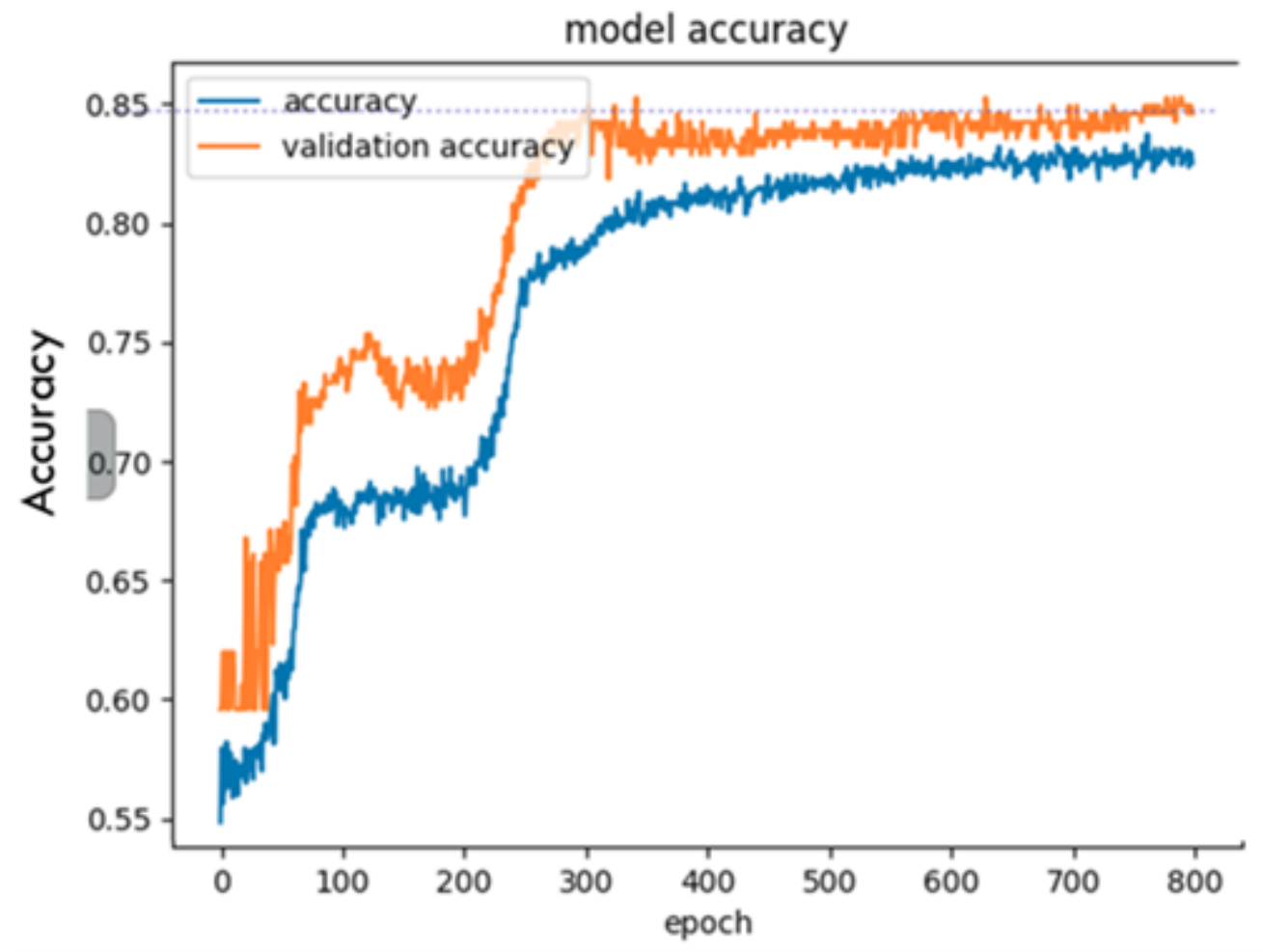

Figure 6

Training and validation accuracy per epoch in finalized ANN model.

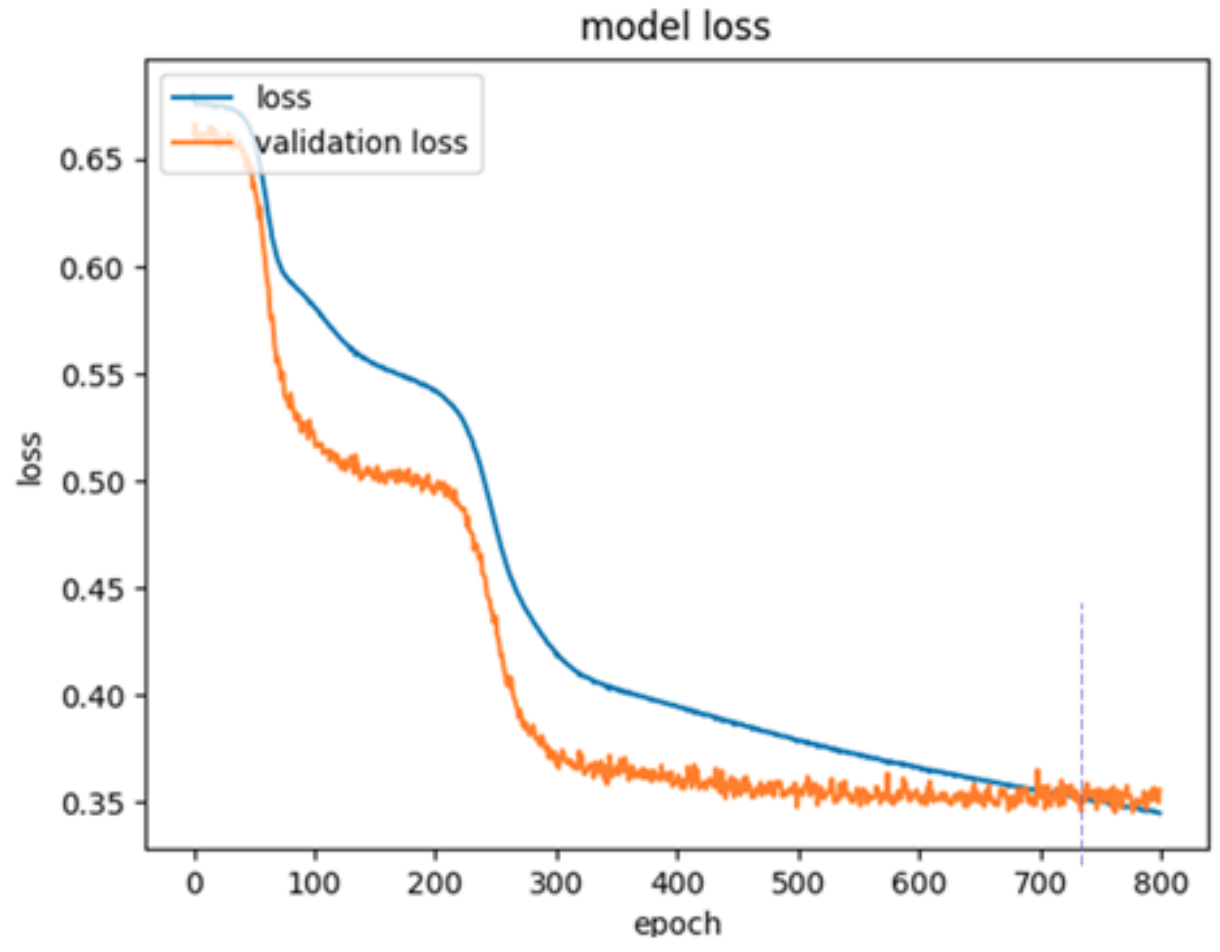

Figure 7

Training and validation loss per epoch in finalized ANN model. 


\section{CNERC AI Welding Parameters Generator}

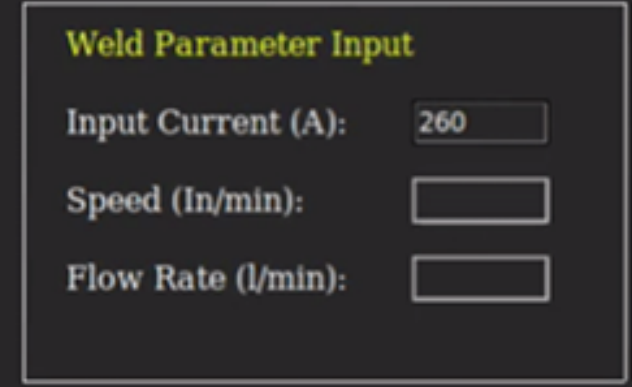

Configuration

Base Metal:

> Material: Steel $>$ Thickness: $6 \mathrm{~mm}$

-> Joint Type: Square Groove Butt Joint

Fillerwire:

$\rightarrow$ Material: Steel $>$ Diameter: $1.2 \mathrm{~mm}$

Protective Gas: MIGSHIELD

Control Pannel

Confirm

Reset

AI System Console

9.9677294e-01 5.0991774e-05]

[AI] Iteration \#2:

Input vector: II $\mathbf{0 . 4 2 6 3 5 2 2 5} \mathbf{0 . 4 2 6 3 5 2 2 5} \mathbf{0 . 4 2 6 3 5 2 2 5}$

Bxit

Help

0.426352250 .426352250 .42635225

$0.426352250 .426352250 .426352250 .4 \xi 035225$

Progress

-

Detected hardware

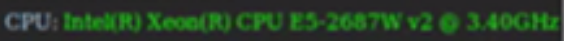

GPUANWIDA GWU | CUDA | cuDNN Supperted $0.42635225 \cdot 2.38365801$

$-2.30621678]]$

(AI) Network Output:I[2.6337969e-01 1.4170235e-01

9.9620706e-01 4.9471855e-05]]

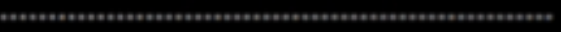

AI Output

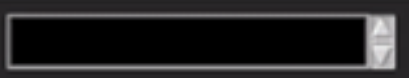

一.

$-\cdot-\cdot$

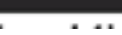

\section{Figure 8}

Al based welding parameters generator. 


\section{CNERC AI Welding Defects Classifier}

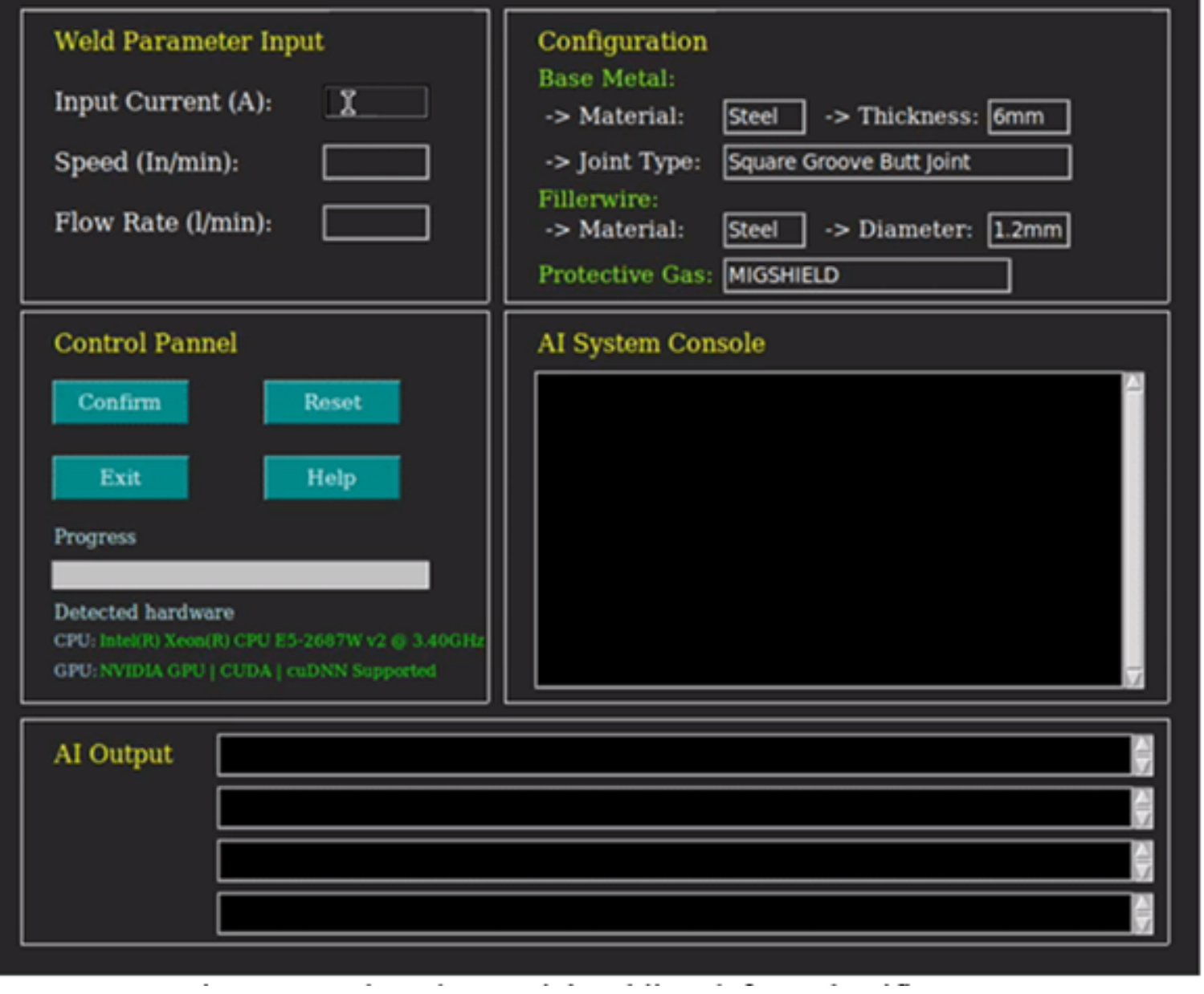

Figure 9

Al based potential welding defects classifier. 